\title{
El Diseño Simplex-Centroide y la Función de Deseabilidad en la optimización de la aceptabilidad sensorial de pan dulce enriquecido con Chenopodium quinoa
}

\section{(The Simplex-Centroid Design and Desirability Function in optimizing the sensory acceptability of sweet bread enriched with Chenopodium quinoa)}

\author{
Raúl Siche ${ }^{1}$, Victor Aredo², Lía Velásquez² ${ }^{\text {, Israel Castillo }}{ }^{1}$
}

\begin{abstract}
Resumen:
El objetivo de este trabajo fue optimizar la aceptabilidad sensorial (AS) de pan dulce (bizcocho andino) elaborado con diferentes porcentajes de harina de trigo $(H T)$, harina de quinua $(H Q)$ y azúcar (A) mediante Diseño Simplex-Centroide (DSC) y Función de Deseabilidad (FD). Se realizaron 10 tratamientos, en los cuales la mezcla de diferentes porcentajes de HT $(28 \%$ $52.5 \%), \mathrm{HQ}(0 \%-24.5 \%)$ y $\mathrm{A}(17.5 \%-42 \%)$ representaron el $70 \%$ de la formulación. La evaluación de la AS fue realizada por triplicado con 20 panelistas consumidores aplicando una escala no estructurada ( 0 a 10). El análisis estadístico del DSC reportó que un modelo cúbico explicó satisfactoriamente el efecto de $H T, H Q$ y $A$ en la $A S\left(p=0.0529 \approx 0.05\right.$ y $\left.R^{2}=94.49 \%\right)$, en la superficie de respuesta se observó que los rangos porcentuales que optimizaron la AS fueron: $35.35 \%-41.97 \%$ de HT, $7.11 \%-11.03 \%$ de HQ y $20.44 \%-24.61 \%$ de A. Mediante FD se determinó que los porcentajes que optimizaron la AS fueron: $40.25 \%$ de HT, $9.05 \%$ de HQ y $20.70 \%$ de $A$.
\end{abstract}

Palabras clave: Diseño de mezclas; evaluación sensorial; bizcocho andino; pan de quinua; aceptabilidad general

\begin{abstract}
:
The aim of this study was to optimize the sensory acceptability (SA) of an andean sweet bread made with different percentages of wheat flour (WF), quinoa flour (QF) and sugar (S) was optimized through Simplex-Centroid Design (SCD) and Desirability Function (DF). Ten treatments were performed, in which the mixture of different percentages of WF $(28 \%-52.5 \%)$, QF (0\% - 24.5\%) and S (17.5\% - 42\%) accounted for $70 \%$ of the dough formula. The evaluation of the SA was conducted in triplicate with 20 consumer panelists of sweet bread, applying an unstructured scale from 0 to 10 . Statistical analysis of SCD reported that a cubic model successfully explained the effect of WF, QF and $S$ on $S A\left(p=0.0529 \approx 0.05\right.$ and $\left.R^{2}=94.49 \%\right)$, analyzing the response surface was observed that the ranges of the percentage that optimize the SA were: $35.35 \%-41.97 \%$ of WF, $7.11 \%-11.03 \%$ QF and $20.44 \%-24.61 \%$ of S. By DF was determined that the percentages that optimize the SA were: $40.25 \%$ of WF, $9.05 \%$ QF and $20.70 \%$ of $S$.
\end{abstract}

Keywords: Mixture design, sensory evaluation, andean sweet bread, quinoa bread, general acceptability

\footnotetext{
1 Universidad Nacional de Trujillo, Trujillo - Perú (rsiche@unitru.edu.pe)

${ }^{2}$ Universidade de São Paulo, Pirassununga SP - Brasil ( \{vj.aredo, lia.vc\} @usp.br )
} 


\section{Introducción}

Las semillas de quinua (Chenopodium quinoa Willd.) han sido usadas tradicionalmente en la región andina para el consumo humano (Repo-Carrasco-Valencia et al., 2003; Berti et al., 2004; Ruiz et al., 2014). En la actualidad, el consumo de quinua se ha expandido a muchas regiones en el mundo, lo cual ha conllevado que la superficie cultivada y la producción total haya sido casi duplicada y triplicada respectivamente en los principales países productores de quinua (Bolivia, Perú y Ecuador) durante el período 1992-2010 (FAOSTAT, 2013). A su vez, su cultivo se ha expandido a países como Estados Unidos, Canadá, Francia, Inglaterra, Suecia, España, Dinamarca, Finlandia, Holanda, Italia, Kenia e India (Medina et al., 2010; FAOSTAT, 2013).

La principal razón de la expansión del consumo y cultivo de la quinua es la valorización de la semilla debido a sus cualidades nutritivas excepcionales; ya sea por su contenido proteico de 918\% (Nowak et al., 2015; Vidueiros et al., 2015; Pineli et al., 2015) con una equilibrada composición de aminoácidos esenciales (Comai et al., 2007) y pocos casos de alergia o intolerancia (Fok et al., 2013); por la presencia de ácidos grasos oleico y linoleico (Álvarez-Jubete et al., 2009); por ser fuente importante de fibra, vitaminas como ácido fólico (Schoenlechner et al., 2010) y vitamina $\mathrm{E}$ (Ryan et al., 2007), minerales como Ca, Mg, Zn y Fe, y antioxidantes como los flavonoides que están vinculados a la prevención de diversas enfermedades (James, 2009; RepoCarrasco-Valencia y Astuhuaman-Serna, 2011).

El pan, como el alimento básico más popular en el mundo, ya sea por su aporte nutricional energético, precio bajo, y sencillez de consumo puede ser enriquecido con ingredientes de alta calidad nutricional y así abastecer la demanda actual de alimentos saludables que hacen frente al aumento de la incidencia de enfermedades ligadas a la mala nutrición (Dewettinck et al., 2008; Sivam et al., 2010; Álvarez-Jubete et al., 2010; Graf et al., 2014). En el caso particular de las comunidades andinas del Perú, los productos de panificación tradicionales como el pan dulce (bizcocho andino) son consumidos masivamente por pobladores y turistas, por lo cual una reformulación que incluya una materia prima altamente disponible como la quinua implicaría mejoras nutricionales, reducción de costo y especialidad del producto.

La adición de quinua ha sido evaluada en productos de panificación (Elgetia et al., 2014; Velásquez et al., 2014; Alencara et al., 2015; Iglesias-Puiga et al., 2015) y en otros productos alimentarios (Mäkinen et al., 2015; Pineli et al., 2015; Caipo et al., 2015), en esos trabajos existe preocupación por la baja aceptabilidad sensorial. Pues, la aceptabilidad sensorial es el principal referente del consumidor en la evaluación de la calidad de un producto, quedando supuesto en su juicio, el cumplimiento de otras características de calidad de tipo físico-químico, nutricional y microbiológico (Aredo et al., 2014; Velásquez et al., 2014; Vásquez-Villalobos et al., 2015a, b).

La optimización de la aceptabilidad sensorial es un paso importante en el desarrollo de nuevos productos, y para alcanzar este objetivo, el Diseño Simplex-Centroide de la Metodología de 
Superficie de Respuesta se ha aplicado con éxito en varias investigaciones (Ricce et al., 2013; Velásquez et al., 2014; Caipo et al., 2015), esta metodología permite la identificación de rangos de las variables que optimizan una respuesta mediante la obtención de una ecuación de respuesta que describe el comportamiento de las variables sin implicar la realización de un conjunto completo de experimentos (Siche et al., 2015; Siche et al., 2016). Además, con la aplicación de la Función de Deseabilidad como herramienta complementaria al Diseño Simplex-Centroide, se pueden identificar valores exactos de las variables que optimizan una respuesta (Siche et al., 2016; Paterakis et al., 2002; Li et al., 2007).

De acuerdo con lo expuesto, el objetivo de este trabajo fue optimizar la aceptabilidad sensorial de un pan dulce preparado con diferentes porcentajes de harina de trigo, harina de quinua y azúcar mediante la aplicación de Diseño Simplex-Centroide de la Metodología de Superficie de Respuesta y Función de Deseabilidad.

\section{Metodología}

\section{Harina de trigo (HT) y harina de quinua (HQ)}

Las semillas de quinua (Chenopodium quinoa wild) variedad Salcedo INIA y las semillas de trigo (Triticum aestivum) variedad Moray, procedieron de la localidad de Huamachuco - Sánchez Carrión (La Libertad / Perú). Las semillas fueron seleccionadas para separar materiales extraños, lavadas con agua potable, desinfectadas con una solución de $5 \mathrm{ml}$ de cloro en 10 litros de agua durante 5 minutos, enjuagadas con agua potable, dejadas reposar para escurrir del exceso de humedad y secadas en secadores solares hasta peso constante. Finalmente, las semillas ingresaron a un proceso de molienda usando un molino de martillos (Mikro-pulverizador, capacidad de $1.5 \mathrm{t}$ / hora, 10 H.P. de potencia) hasta alcanzar granulometrías comunes en harinas integrales, adicionalmente las partículas groseras correspondientes al salvado en la harina de trigo fueron retiradas por cernido.

\section{Formulación y elaboración del pan dulce}

Los tratamientos del diseño experimental se pueden observar en la Tabla 1, en los cuales los tres principales ingredientes del pan dulce constituyeron el $70 \%$ del total de la fórmula y variaron entre los siguientes porcentajes: harina de trigo (HT) $(28 \%$ - $52.5 \%)$, harina de quinua (HQ) $(0 \%$ $24.5 \%)$ y azúcar (A) (17.5\% - 42\%); mientras que los demás ingredientes permanecieron constantes en los siguientes porcentajes: manteca vegetal (4\%), yema de huevo (7\%), levadura seca comprimida (Saccharomyces cerevisiae, Fleischmann, Perú) (1\%), agua filtrada (18\%). La incorporación de poca agua en la formulación (18\%) le confiere al producto la consistencia típica de un bizcocho andino elaborado en Huamachuco, La Libertad, Perú (3269 m.s.n.m., $12{ }^{\circ} \mathrm{C}$ de temperatura promedio). Además, el proceso que a continuación se indica, también es el mismo que utilizan en esta zona andina del Perú. El primer paso fue la mezcla de las harinas de trigo y 
quinua, azúcar y levadura para asegurar la homogeneidad de estos ingredientes sólidos en la masa, luego se agregó agua y se mezcló hasta observar una masa hidratada, para así adicionar los ingredientes restantes. El amasado fue manual por aproximadamente 15 minutos y se obtuvo una masa homogénea, compacta y elástica. La masa reposó 30 minutos a $12{ }^{\circ} \mathrm{C}$ (temperatura ambiente del área de elaboración) con la finalidad de que la levadura induzca el crecimiento de la masa y aporte características sensoriales propias del producto. Luego, se realizó un formado manual de porciones de masa de 60 gramos aproximadamente y se dejó reposar para una segunda fermentación por 45 minutos. Posteriormente, ingresaron a un proceso de horneado en un horno tradicional andino a leña, durante 30 minutos a $120 \pm 10 \stackrel{\circ}{\circ}$ (temperatura monitoreada con termómetro de mercurio y controlada manualmente mediante adición o retiro de leña) hasta que el pan dulce adquirió un color dorado característico, producto de la caramelización de azúcares, y enfriamiento a temperatura ambiente por 60 minutos. Finalmente, los panes dulces fueron envasados en bolsas de polipropileno y almacenados en un lugar fresco y seco.

\section{Evaluación sensorial}

La evaluación sensorial del producto se realizó en el Laboratorio de Tecnología de los Productos Agroindustriales de la Facultad de Ciencias Agropecuarias de la Universidad Nacional de Trujillo Sede Huamachuco. La aceptabilidad sensorial (AS) fue evaluada por 20 panelistas no entrenados consumidores frecuentes de pan dulce de la localidad de Huamachuco, previamente seleccionados; y fue realizada por triplicado para incrementar el número de evaluaciones a 60 , debido al limitado número de consumidores con predisposición a la participación en este tipo de investigaciones. Los panelistas recibieron un vaso con agua, muestras de pan dulce de cada tratamiento, y una ficha de evaluación sensorial en la cual utilizaron una escala hedónica no estructurada (recta continua de $10 \mathrm{~cm}$ donde 0 equivale a "me desagrada mucho", 5 a "me es indiferente" y 10 "me agrada mucho") para expresar la intensidad de la AS en un valor de tipo continuo; esta escala ha demostrado ser más amigable con el consumidor, adquiriendo información sensorial representativa para el modelamiento (Aredo et al., 2014; Vásquez-Villalobos et al., 2015a).

\section{Optimización estadística}

El Diseño Simplex-Centroide (DSC) de la Metodología de Superficie de Respuesta consiste en un triángulo que representa todo el universo de posibilidades de mezcla de los tres ingredientes evaluados ( $\mathrm{HT}, \mathrm{HQ}$ y A). El análisis estadístico de regresión múltiple para evaluar el efecto de los ingredientes en la AS del pan dulce fue realizado usando el programa Statistica versión 6.0 (Statsoft USA, 2004) y requirió de un análisis de varianza $(\alpha=0.05)$ de modelos de regresión lineal, cuadrático y cúbico, para escoger el modelo más significativo $(p<0.05)$ y de mejor ajuste $\left(R^{2}>0.85\right)$ con el cual se construyó la superficie de respuesta para determinar los rangos de los tres ingredientes que optimizan la AS. Con la misma data, como complemento al DSC se 
construyeron funciones de deseabilidad (FD) cuadráticas que determinaron el valor porcentual de cada ingrediente (HT, HQ y A) que optimizó la AS.

\section{Resultados y discusión}

La AS de los 10 tratamientos del pan dulce elaborado con porcentajes diferentes de HT, HQ y A se muestra en la Tabla 1, esta varió en el rango de 3.24 a 9.14 de la escala de evaluación de 0 a 10, lo cual sugiere que los niveles porcentuales de $H T, H Q$ y $A$ permitieron una exploración completa de la AS del pan dulce, abarcando valores de AS mayores a 7.5 clasificados como "AS muy alta" por Aredo et al. (2014) para escalas no estructuradas. Entre los tratamientos, el T8 presentó mayor AS (9.14) al estar compuesto por una mezcla $44.33 \%$ de HT, $4.08 \%$ de HQ y $21.58 \%$ de azúcar, seguido del T9 y T7 con una AS de 8.86 y 8.30 , respectivamente. La composición de estos tratamientos evidenció que en general los niveles intermedios de $\mathrm{HT}, \mathrm{HQ}$ y A generan mayor AS, es decir que la interacción de los ingredientes es positiva para la AS, mientras que individualmente los ingredientes $(H T, H Q$ y $A)$ generan una AS baja, por ejemplo, los tratamientos T3, T5, T6 y T10 tuvieron una AS baja posiblemente causada por el contenido alto de A (29.75\%-42\%). Los comportamientos mencionados se discuten más adelante al interpretar la superficie de respuesta (Figura 1).

Tabla 1. Variables independientes y aceptabilidad sensorial de las muestras.

\begin{tabular}{|c|c|c|c|c|c|c|c|}
\hline \multirow{3}{*}{$\mathrm{T}$} & \multicolumn{6}{|c|}{ Variables independientes } & \multirow{3}{*}{$\begin{array}{c}\text { Respuesta } \\
\text { AS }^{*}\end{array}$} \\
\hline & \multicolumn{3}{|c|}{ Valor codificado } & \multicolumn{3}{|c|}{ Valor real (\%) } & \\
\hline & $\mathrm{HT}$ & $\mathrm{HQ}$ & $A$ & HT & $\mathrm{HQ}$ & $A$ & \\
\hline T1 & 1 & 0 & 0 & 52.5 & 0 & 17.5 & $7.95 \pm 0.93$ \\
\hline $\mathrm{T} 2$ & 0 & 1 & 0 & 28 & 24.5 & 17.5 & $4.20 \pm 1.21$ \\
\hline T3 & 0 & 0 & 1 & 28 & 0 & 42 & $3.24 \pm 1.16$ \\
\hline T4 & $1 / 2$ & $1 / 2$ & 0 & 40.25 & 12.25 & 17.5 & $8.13 \pm 0.84$ \\
\hline T5 & $1 / 2$ & 0 & $1 / 2$ & 40.25 & 0 & 29.75 & $4.63 \pm 1.03$ \\
\hline T6 & 0 & $1 / 2$ & $1 / 2$ & 28 & 12.25 & 29.75 & $6.11 \pm 0.91$ \\
\hline T7 & $1 / 3$ & $1 / 3$ & $1 / 3$ & 36.17 & 8.17 & 25.67 & $8.30 \pm 0.81$ \\
\hline T8 & $2 / 3$ & $1 / 6$ & $1 / 6$ & 44.33 & 4.08 & 21.58 & $9.14 \pm 0.72$ \\
\hline 79 & $1 / 6$ & $2 / 3$ & $1 / 6$ & 32.08 & 16.33 & 21.58 & $8.86 \pm 0.76$ \\
\hline $\mathrm{T} 10$ & $1 / 6$ & $1 / 6$ & $2 / 3$ & 32.08 & 4.08 & 33.83 & $6.63 \pm 0.93$ \\
\hline
\end{tabular}

$\mathrm{T}=$ Tratamiento; $\mathrm{HT}=$ Harina de trigo; $\mathrm{HQ}=$ Harina de quinua; $\mathrm{A}=$ Azúcar; $\mathrm{AS}=$ Aceptabilidad sensorial .

* AS expresada en valor promedio \pm desviación estándar, $n=3$.

Sivam et al. (2010) y Das et al. (2012) señalan que la adición de un nuevo ingrediente a productos de panificación puede cambiar las propiedades tecnológicas y nutricionales; así Morita et al. (2001) e Iglesias-Puiga et al. (2015) compararon un pan simple con un pan con HQ y observaron que con el uso de quinua, componentes como los lípidos, fibra dietética y cenizas aumentan, el volumen específico disminuye, la miga se vuelve más densa y compacta por la menor presencia de gluten de la harina de trigo, y el color varía (en la corteza y miga) debido a los carotenoides, clorofila y lignina provenientes de la quinua (Ruffino et al., 2010). Dado que los cambios tecnológicos y nutricionales causados por el uso HQ en pan son conocidos y han sido explicados 
en otras investigaciones, este trabajo se enfocó en optimizar la AS como indicador general de la calidad sensorial del producto, ya que la quinua puede generar disminución en la calidad sensorial del pan (Iglesias-Puiga et al., 2015).

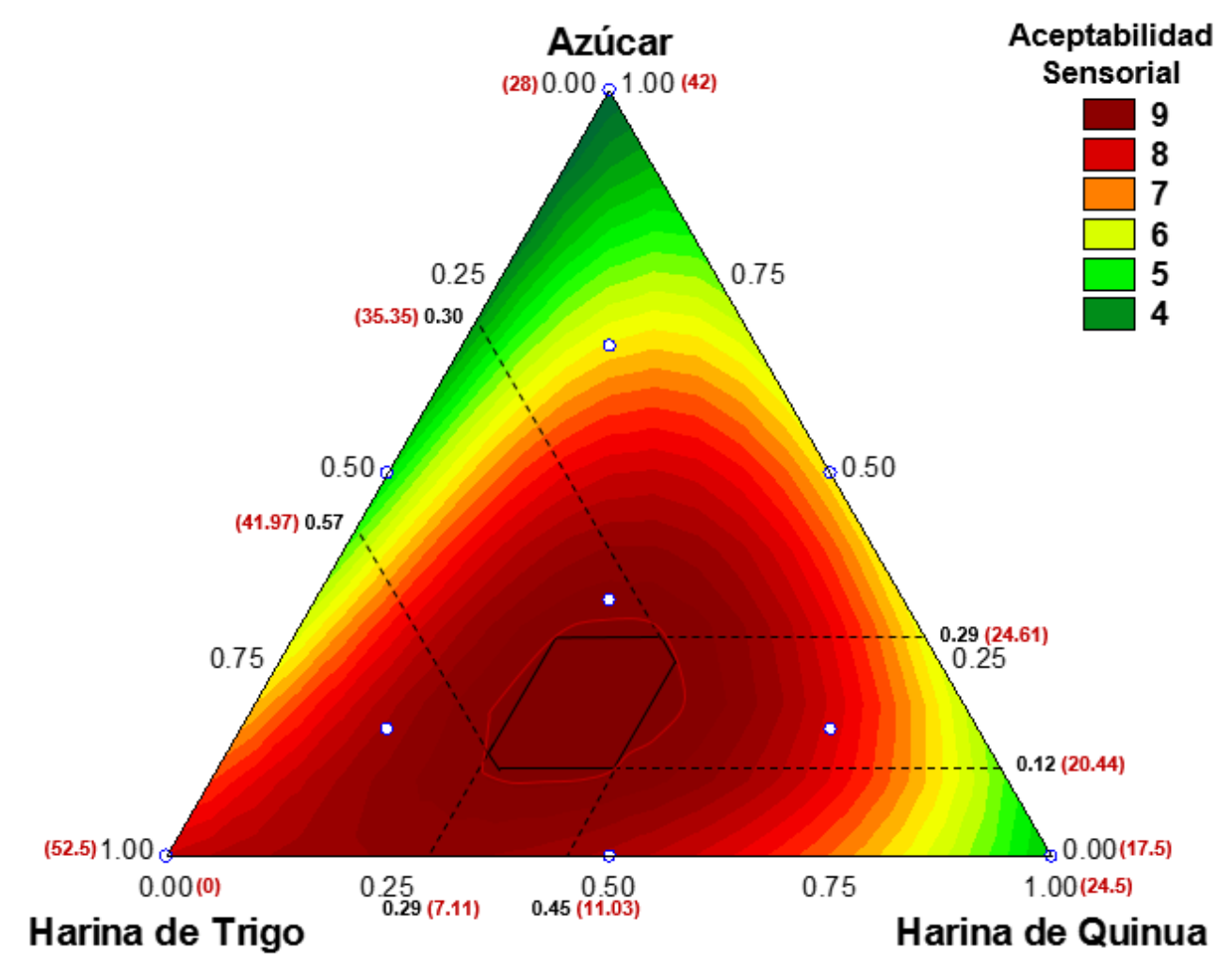

Figura 1. Gráfico de contorno de la superficie de respuesta de la aceptabilidad sensorial.

\section{Optimización por Diseño Simplex-Centroide (DSC)}

En la optimización por DSC, el análisis de varianza de los modelos de regresión que explican la AS en base a los porcentajes de HT, HQ y A, reportó que los modelos de tipo lineal, cuadrático y cúbico tienen valores $p$ de $0.1574,0.0816$ y 0.0529 , y coeficientes de determinación $R^{2}(\%)$ de 41.04, 85.24 y 94.49, respectivamente. El modelo cúbico fue elegido por tener el efecto estadístico significativo más próximo al valor del nivel de confianza $(p=0.0529 \approx 0,05)$ y por poseer un elevado coeficiente de determinación $\mathrm{R}^{2}(94.49 \%>85 \%)$. El modelo fue expresado de la siguiente manera:

Aceptabilidad sensorial $=0.2281^{*}(\mathrm{HT})+1.3181^{*}(\mathrm{HQ})+0.0974^{*}(\mathrm{~A})-0.0585^{*}(\mathrm{HT})^{\star}(\mathrm{HQ})-$ $0.0062^{*}(H T)^{\star}(A)-0.1003^{*}(H Q)^{*}(A)+0.0042^{*}(H T)^{*}(H Q)^{*}(A)\left(R^{2}=94,49 \% ; p=\right.$ $0.0529)$

Donde :

$$
H T=\text { Harina de trigo; } H Q=\text { Harina de quinua; } A \text { = Azúcar }
$$

Los signos positivos en los coeficientes del modelo sugieren sinergia, indicando que los efectos combinados o de interacción entre los ingredientes generan respuestas mayores cuando se mezclan. Asimismo, el signo negativo implica antagonismo entre los ingredientes, es decir que se 
producen respuestas menores (Cho et al., 2009). De acuerdo con Velásquez et al. (2014) en un Diseño Simplex-Centroide las sinergias o antagonismos son notorios cuando para explicar la respuesta se eligen modelos cuadráticos o cúbicos (cuyas ecuaciones cuentan con elementos de interacción entre las variables), de esta misma manera en este trabajo al evaluar los coeficientes del modelo cúbico se observó que los tres ingredientes individualmente influyen positivamente del mismo modo que su interacción total en la AS, mientras que las interacciones duales no lo hacen.

Definido el modelo, se realizó la interpretación gráfica de la superficie de respuesta en el formato de gráfico de contornos para conocer los rangos de los porcentajes de HT, HQ y A que optimizan la AS en el pan dulce. En la Figura 1, se puede comprobar que los ingredientes individualmente no generan una AS tan alta como cuando existe interacción entre ingredientes. La región de interés que indica la máxima AS está representada por la zona de color rojo oscuro, mas con el fin de asegurar la reproducibilidad de resultados óptimos, la región de interés fue redefinida como un polígono inscrito en la región inicial de interés construida por rectas paralelas a los lados del triángulo que representan a las variables. Con la lectura de esta región de interés, se observa que los rangos porcentuales de $\mathrm{HT}, \mathrm{HQ}$ y A que optimizan la AS son los siguientes: $35.35 \%-41.97 \%$ de $\mathrm{HT}, 7.11 \%-11.03 \%$ de HQ y $20.44 \%-24.61 \%$ de $\mathrm{A}$.

\section{Optimización por Función de Deseabilidad (FD)}

Los valores porcentuales de cada ingrediente que optimizan la AS del pan dulce determinados por FD son los siguientes: $40.25 \%$ de HT, $9.05 \%$ de HQ y $20.70 \%$ de A (Figura 2). Estos valores pertenecen a los rangos determinados por DSC, y garantizan que un pan dulce elaborado con esos valores porcentuales de HT, HQ y A tendrá una alta aceptabilidad (AS>9). La utilización de los valores determinados por FD o los rangos determinados por DSC que optimizan la AS van a depender de la escala productiva, ya que por ejemplo generalmente en la industria de alimentos se prefiere usar rangos de variables en vez de valores exactos para agilizar los procesos.

EI DSC y FD optimizaron la AS del pan dulce con procedimientos prácticos y simples, lo que resulta particularmente útil para la validación de resultados en otro público objetivo o incluso dentro del mismo, ya que los mercados de consumidores tienden a cambiar constantemente de gustos y preferencias, demandando nuevos productos de alta calidad sensorial.

En futuros trabajos se recomienda aplicar DSC y FD en la optimización de la AS de productos de panificación que incorporen $\mathrm{HQ}$ libre de antinutrientes que afectan la biodisponibilidad de minerales, proteínas y oligoelementos. También DSC y FD podrían ser usadas en la optimización de los procesos que eliminen antinutrientes de la quinua, ya que actualmente, las saponinas pueden ser eliminadas por lavado o maceración de los granos en agua (Pineli et al., 2015); el ácido fítico o sus sales (fitatos) pueden ser eliminadas con procesos enzimáticos de aplicación de fitasas exógenas (Garcia-Mantrana et al., 2014; Iglesias-Puiga et al., 2015) debido a que bajo 
procesamiento convencional de panificación difícilmente hay degradación de estos compuestos (Hager et al., 2012).
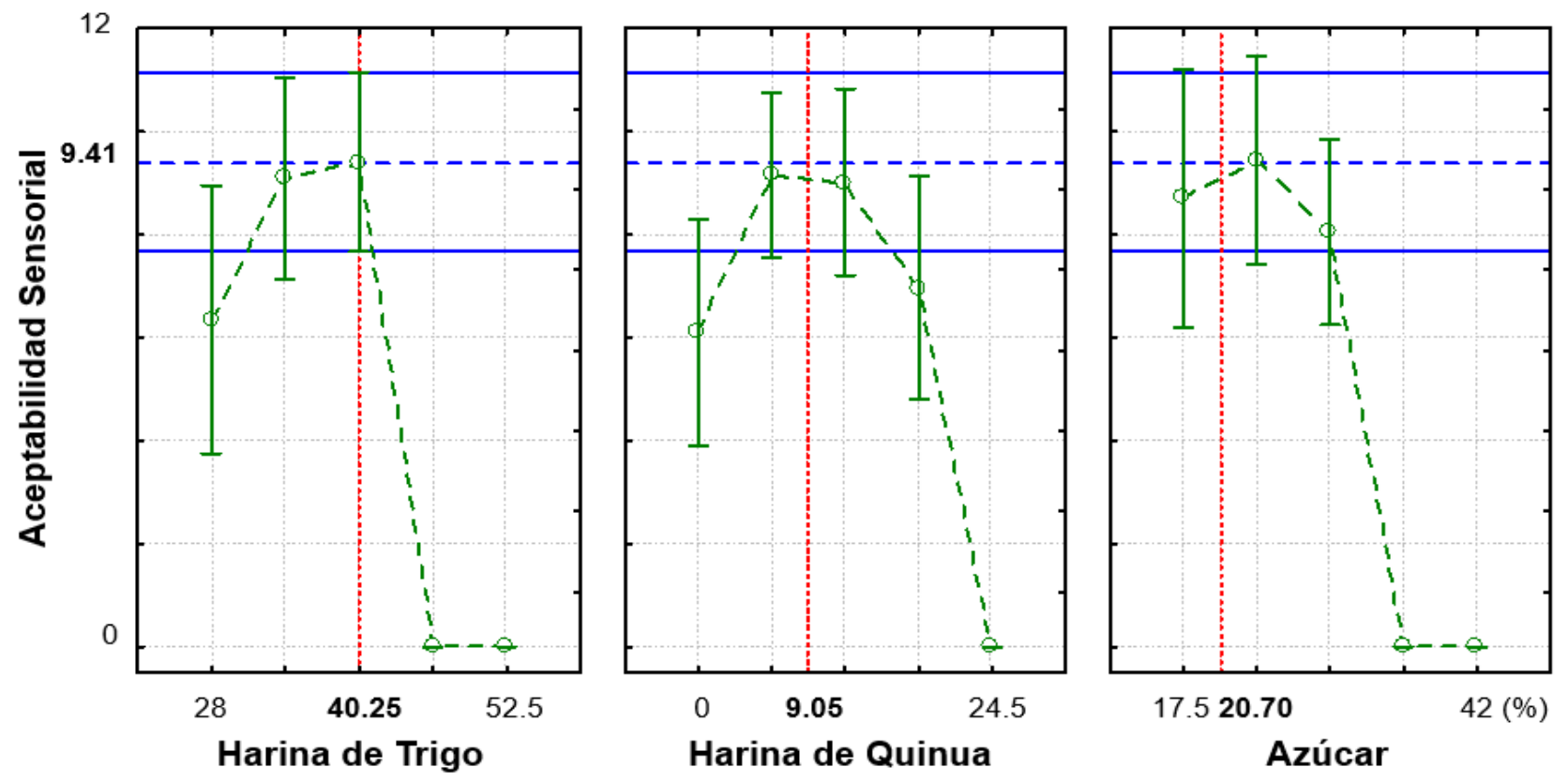

Figura 2. Optimización de la aceptabilidad sensorial mediante función de deseabilidad.

\section{Conclusiones y recomendaciones}

El Diseño Simplex-Centroide (DSC) y Función de Deseabilidad (FD) optimizaron la aceptabilidad sensorial (AS) de un pan dulce (o bizcocho andino), en el cual se variaron los porcentajes de harina de trigo $(\mathrm{HT})(28 \%-52.5 \%)$, harina de quinua $(\mathrm{HQ})(0 \%-24.5 \%)$ y azúcar $(\mathrm{A})(17.5 \%$ $42 \%$ ), alcanzando valores de AS mayores a 9, en una escala de 0 a 10.

Mediante DSC se determinó que los rangos porcentuales que optimizaron la AS fueron: 35.35\% $41.97 \%$ de HT, $7.11 \%$ - $11.03 \%$ de $\mathrm{HQ}$ y $20.44 \%$ - $24.61 \%$ de A; y con FD se determinó que los porcentajes que optimizaron la AS fueron: $40.25 \%$ de $\mathrm{HT}, 9.05 \%$ de $\mathrm{HQ}$ y $20.70 \%$ de $\mathrm{A}$. Estos resultados contribuyen en el desarrollo de un pan dulce de alta aceptabilidad como producto de mejor calidad nutricional debido a la incorporación de $\mathrm{HQ}$ en su formulación.

El procedimiento de optimización empleado en este trabajo puede ser de utilidad para estudiar la aceptabilidad sensorial de otros productos de panadería.

\section{Agradecimientos}

Víctor Aredo y Lía Velásquez agradecen al "Ministerio de Educación del Perú" por las becas de estudios de posgrado otorgadas por medio del "Programa Nacional de Becas y Crédito Educativo" (PRONABEC). Raúl Siche agradece al Fondo Cánon UNT (PIC-2/2013) por el soporte financiero de esta investigación. 


\section{Bibliografía}

Alencara, N. M. M., Steela, C. J., Alvimb, I. D., Moraisa, E. C., \& Bolinia, H. M. A. (2015). Addition of quinoa and amaranth flour in gluten-free breads: Temporal profile and instrumental analysis. LWT - Food Science and Technology, 62 (2), 1011-1018.

Álvarez-Jubete, L., Arendt, E. K., \& Gallagher, E. (2009). Nutritive value and chemical composition of pseudocereals as gluten-free ingredients. International Journal of Food Science and Nutrition, 60, 240-257.

Álvarez-Jubete, L., Arendt, E. K., \& Gallagher, E. (2010). Nutritive value of pseudocereals and their increasing use as functional gluten-free ingredients. Trends in Food Science \& Technology, $21,106-113$.

Aredo, V., Velásquez, L., Narro, O., \& Domínguez, R. (2014). Response Surface Method and Fuzzy Modelling in the development of cookies with chia seed (Salvia hispánica L.). Agroindustrial Science, 4(1), 27 - 34.

Berti, C., Ballabio, C., Restani, P., Porrini, M., Bonomi, F., \& lametti, S. (2004). Immunochemical and molecular properties of proteins in Chenopodium quinoa. Cereal chemistry, 81(2), 275277.

Caipo, Y., Gutiérrez, A., \& Julca, A. (2015). Optimization by mixtures design of the acceptability of an energy bar from quinoa (Chenopodium quinoa), amaranth (Amaranthus caudatus) and cañihua (Chenopodium pallidicaule) evaluated in children. Agroindustrial Science, 5(1), 6167.

Cho, R., Shin, S., Choi, Y., \& Kovach, J. (2009). Development of a multidisciplinary optimization process for designing optimal pharmaceutical formulations with constrained experimental regions. International Journal of Advanced Manufacturing Technology, 44, 9-10.

Comai, S., Bertazzo, A., Bailoni, L., Zancato, M., Costa, C., \& Allegri. G. (2007). The content of proteic and nonproteic (free and protein-bound) tryptophan in quinoa and cereal flours. Food Chemistry, 100, 1350-1355.

Das, L., Raychaudhuri, U., \& Chakraborty, R. (2012). Supplementation of common white bread by coriander leaf powder. Food Science and Biotechnology, 21, 425-433.

Dewettinck, K., Van Bockstaele, F., Kühne, B., Van de Walle, D., Courtens, T.M., \& Gellynck, X. (2008). Nutritional value of bread: Influence of processing, food interaction and consumer perception. Journal of Cereal Science, 48, 243-257. 
Elgetia, D., Nordlohneb, S. D., Föstea, M., Besla, M., Lindenb, M. H., Heinzb, V., Jeklea, M., \& Beckera, T. (2014). Volume and texture improvement of gluten-free bread using quinoa white flour. Journal of Cereal Science, 59 (1), 41-47

FAOSTAT. (2013). What is quinoa? Distribution and production. International Year of Quinoa Secretariat Food and Agriculture Organization of the United Nations Regional Office for Latin America and the Caribbean, Santiago, Chile. Recuperado de http://www.fao.org/quinoa2013/what-is-quinoa/distribution-and-production/en/

Fok, J. S., Hissaria, P., Giri, P., Heddle, R., \& Smith, W. (2013). Anaphylaxis to quinoa. Annals of Allergy. Asthma \& Immunology, 110, 60-61.

Garcia-Mantrana, I., Monedero, V., \& Haros, M. (2014). Application of phytases from bifidobacteria in the development of cereal-based products with amaranth. European Food Research and Technology, 238, 853-862

Graf, B.L., Poulev, A., Kuhn, P., Grace, M.H., Lila, M.A., \& Raskin, I. (2014). Quinoa seeds leach phytoecdysteroids and other compounds with anti-diabetic properties. Food Chemistry, 163, $178-185$.

Hager, A-S., Wolter, A., Jacob, F., Zannini, E., \& Arendt, E. K. (2012). Nutritional properties and ultra-structure of commercial gluten free flours from different botanical sources compared to wheat flours. Journal of Cereal Science, 56, 239-247.

Iglesias-Puiga, E., Monederob, V., \& Harosa, M. (2015). Bread with whole quinoa flour and bifidobacterial phytases increases dietary mineral intake and bioavailability. LWT - Food Science and Technology, 60 (1), 71-77.

James, L. E. A. (2009). Quinoa (Chenopodium quinoa Willd.): Composition, chemistry, nutritional, and functional properties. Advances in Food and Nutrition Research, 58, 1-31.

Li, J., Ma, C., Ma, Y., Li, Y., Zhou, W., \& Xu, P. (2007). Medium optimization by combination of response surface methodology and desirability function: an application in glutamine production. Applied microbiology and biotechnology, 74(3), 563-571.

Mäkinen, O. E., Uniacke-Lowe, T., O'Mahony, J. A., \& Arendt, E. K. (2015). Physicochemical and acid gelation properties of commercial UHT-treated plant-based milk substitutes and lactose free bovine milk. Food Chemistry, 168, 630-638.

Medina, W., Skurtys, O., \& Aguilera, J. M. (2010). Study on image analysis application for identification quinoa seeds (Chenopodium quinoa Willd) geographical provenance. LWT Food Science and Technology, 43, 238-246. 
Morita, N., Hirata, C., Park, S. H., \& Mitsunaga, T. (2001). Quinoa flour as a new foodstuff for improving dough and bread. Journal of Applied Glycoscience, 48, 263-270.

Nowak, V., Du, J., \& Charrondière, U. R. (2015). Assessment of the nutritional composition of quinoa (Chenopodium quinoa Willd.). Food Chemistry, 193, 47-54.

Paterakis, P. G., Korakianiti, E. S., Dallas, P. P., \& Rekkas, D. M. (2002). Evaluation and simultaneous optimization of some pellets characteristics using a 33 factorial design and the desirability function. International Journal of Pharmaceutics, 248(1), 51-60.

Pinelia, L. L. O., Botelhoa, R. B. A., Zandonadia, R. P., Solorzanob, J. L., Oliveirab, G. T., Reisc, C. E. G., \& Teixeira, D. S. (2015). Low glycemic index and increased protein content in a novel quinoa milk. LWT - Food Science and Technology, 63 (2), 1261-1267.

Repo-Carrasco-Valencia, R. A. M., \& Astuhuaman-Serna. L. (2011). Quinoa (Chenopodium quinoa, Willd.) as a source of dietary fiber and other functional components. Ciência $e$ Tecnologia de Alimentos, 31, 225-230.

Repo-Carrasco-Valencia, R. A. M., Espinoza, C., \& Jacobse. S-E. (2003). Nutritional value and use of the Andean crops quinoa (Chenopodium quinoa) and Kañiwa (Chenopodium pallidicaule). Food Reviews International, 19, 179-189.

Ricce, C., Leyva, M., Medina, I., Miranda, J., Saldarriaga, L., Rodriguez, J., \& Siche, R. (2013). Using waste of La Libertad agroindustry in developing a bread. Agroindustrial Science, 3(1), $41-46$.

Ruffino, A. M. C., Rosa, M., Hilal, M., González, J. A., \& Prado, F. E. (2010). The role of cotyledon metabolism in the establishment of quinoa (Chenopodium quinoa) seedlings growing under salinity. Plant Soil, 326, 213-224.

Ruiz, K. B., Biondi, S., Oses, R., Acuña-Rodríguez, I. S., Antognoni, F., Martinez-Mosqueira, E. A., Coulibaly, A., Canahua-Murillo, A., Pinto, M., Zurita-Silva, A., Bazile, D., Jacobsen, S. E., \& Molina-Montenegro, M. A. (2014). Quinoa biodiversity and sustainability for food security under climate change. Agronomy for Sustainable Development, 34, 349-359.

Ryan, E., Galvin, K., O'Connor, T., Maguire, A., \& O'Brien, N. (2007). Phytosterol, squalene, tocopherol content and fatty acid profile of selected seeds, grains, and legumes. Plant Foods for Human Nutrition, 62(3), 85-91.

Schoenlechner, R., Wendner, M., Siebenhandl-Ehn, S., \& Berghofer, E. (2010). Pseudocereals as alternative sources for high folate content in staple foods. Journal of Cereal Science, 52, 475-479. 
Siche, R., Arteaga, H., Saldaña, E., \& Vieira, T. M. F. S. (2016). Antioxidant Capacity of Binary and Ternary Mixtures of Orange, Grape, and Starfruit Juices. Current Nutrition \& Food Science, 12(1), 65-71.

Siche, R., Falguera, V., \& Ibarz, A. (2015). Use of response surface methodology to describe the combined effect of temperature and fiber on the rheological properties of orange juice. Journal of Texture Studies, 46(2), 67-73.

Sivam, A. S., Sun-Waterhouse, D., Quek, S., \& Perera, C. O. (2010). Properties of bread dough with added fiber polysaccharides and phenolic antioxidants: A review. Journal of Food Science, 75: 163-174.

Vásquez-Villalobos, V., Angulo, J. V., \& Reyna, E. M. (2015a). New method for determining sensory shelf life using fuzzy logic: canned marinated artichoke hearts (Cynara scolymus L.) case. Scientia Agropecuaria, 6(2), 99-109.

Vásquez-Villalobos, V., Aredo, V., Velásquez, L., \& Lázaro, M. (2015b). Physicochemical properties and sensory acceptability of goat's milk fruit yogurts with mango and banana using accelerated testing. Scientia Agropecuaria, 6(3), 177-189.

Velásquez, L., Aredo, V., Caipo, Y. \& Paredes, E. (2014). Optimization by mixtures design of the acceptability of an enriched cookie with quinoa (Chenopodium quinoa), soybean (Glycine max) and cocoa (Theobroma cacao L.). Agroindustrial Science, 4(1), 35 - 42.

Vidueiros, S. M., Curti, R. N., Dyner, L. M., Binaghi, M. J., Peterson, G., Bertero, H. D., \& Pallaro, A. N. (2015). Diversity and interrelationships in nutritional traits in cultivated quinoa (Chenopodium quinoa Willd.) from Northwest Argentina. Journal of Cereal Science, 62, 8793. 